\title{
Three-dimensional printing of bioceramic-induced macrophage exosomes: immunomodulation and osteogenesis/angiogenesis
}

\author{
Yuhua Sun ${ }^{1}$, Bingjun Zhang ${ }^{1}$, Dong Zhai ${ }^{1,2}$ and Chengtie Wu (D) ${ }^{1,2}$
}

\begin{abstract}
Exosomes have attracted increasing attention in tissue regeneration and repair due to their roles in intercellular communication. Developing a customized delivery system is key to exosome-based regenerative therapeutics. Bioceramics play an important role in the immunomodulation of macrophages. Here, three-dimensional (3D) printing was applied to construct porous scaffolds with $\beta$-tricalcium phosphate ( $\beta$-TCP) bioceramic-induced macrophage exosomes (BC-Exos). The three-dimensional-printed BC-Exo scaffolds, exhibiting a predefined structure and persistent release of exosomes, displayed distinct immunomodulatory effects and improved osteogenesis/angiogenesis. The BCExos in the printed scaffolds modulated macrophage polarization and the expression of chemokines for the recruitment of bone marrow mesenchymal stem cells (BMSCs) and endothelial cells. Scaffolds with BC-Exos from macrophages with a mixed phenotype significantly enhanced the osteogenic differentiation and immunosuppression of BMSCs and improved the angiogenic activity of human umbilical vein endothelial cells in vitro. For the potential mechanism, $\beta$-TCP bioceramics have an important effect on the immunomodulation of macrophages by regulating gene expression, increasing exosome production, and altering exosomal miRNA cargos, thereby affecting the paracrine effects of BC-Exos on immunomodulation and osteogenesis/angiogenesis. This study suggests that 3D printing of bioceramic-induced macrophage exosomes may be a useful strategy for tissue engineering and regenerative medicine.
\end{abstract}

\section{Introduction}

In the past few decades, tissue engineering technology has become an effective strategy for repairing lost or damaged tissue. Classically, three key elements are involved in tissue engineering: growth factors, cells, and scaffolds, which are used either singly or in combination in efforts to replace functional tissues. However, despite the reported advantages, the clinical use of growth factors and cells still faces challenges, such as biobanking, production costs, safety, and cryopreservation. In recent

\footnotetext{
Correspondence: Chengtie Wu (chengtiewu@mail.sic.ac.cn)

${ }^{1}$ State Key Laboratory of High Performance Ceramics and Superfine

Microstructure, Shanghai Institute of Ceramics Chinese Academy of Sciences, 1295 Dingxi Road, Shanghai 200050, People's Republic of China

${ }^{2}$ Center of Materials Science and Optoelectronics Engineering, University of Chinese Academy of Sciences, 19A Yuquan Road, Beijing 100049, People's Republic of China
}

years, exosomes, as a class of extracellular vesicles (EVs) of approximately $100 \mathrm{~nm}$ in diameter, have received much attention due to their capacity to transfer proteins, nucleic acids, and lipids, thereby mediating cell-to-cell communication. Since exosomes contain parent cell-specific molecules in the cargo and on the membrane, these vesicles are recognized as potential candidates for the repair of damaged tissue and regulation of body immunity. ${ }^{1,2}$ Compared with the parent cells and growth factors, exosomes are more stable, better stored, and easier to access and control due to their excellent characteristics, including the nanosize, the lipid bilayer membrane, which can protect their functional components from lysosomeinduced degradation, and a subcellular structure free from ethical limits. Exosomes also have low immunogenicity and a reduced potential risk of ectopic engraftment. ${ }^{3}$ 
Consequently, exosomes are considered an alternative to conventional cell therapy, representing a potential cellfree strategy for tissue regeneration. However, exosomebased regenerative therapeutics also present challenges, such as the short lifetime of exosomes in injured tissue due to rapid clearance by the innate immune system in vivo. ${ }^{4}$ As tissue regeneration usually takes a long time and the viability of free exosomes cannot be maintained, multiple administrations are always necessary to realize the regenerative effect. This phenomenon undoubtedly complicates exosome application and increases the suffering of hosts. Controlled release of exosomes from preformed hydrogels resulted in superior regeneration compared with a single dose of the same total concentration of exosomes. ${ }^{5}$ Hydrogels can increase the retention and stability of exosomes in local tissues and simultaneously provide a temporary extracellular matrix for cell growth. Currently, hydrogels for exosome delivery are mainly prepared via freeze-drying, injection molding, bulk crosslinking, or self-assembly technologies. However, these materials display common drawbacks, such as an uncontrollable structure and lack of macropores, which are not conducive to the continuous growth of tissue. Three-dimensional (3D) printing, also known as additive manufacturing, enables the precise control of manufacturing processes and fabrication of tissue constructs of predefined architecture and shapes. This method has been widely used in the preparation of porous scaffolds for bone tissue engineering. ${ }^{6}$ Three-dimensional printing of hydrogels can combine the advantages of both additive manufacturing and hydrogel materials. Hence, in this work, 3D printing was proposed to construct a biocompatible porous hydrogel scaffold for the long-term release of nanosized exosomes and exosome-mediated paracrine effects.

In bone homeostasis, exosomes have been verified to be key regulators of osteogenesis, osteoclastogenesis, and endochondral mineralization by transferring exosomalspecific proteins, miRNAs and $\mathrm{Ca}^{2+} \cdot{ }^{7,8}$ While numerous investigations have focused on the role of stem cell-derived exosomes in bone regenerative medicine, knowledge of the regenerative potential of immune cellderived exosomes is limited. As important effector cells in the immune response, macrophages (MФs) are known to have critical roles in inflammation and tissue healing. ${ }^{9}$ EVs derived from macrophages were shown to improve the inflammatory microenvironment for osteogenic differentiation and vascular repair. ${ }^{10,11}$ Since exosomes contain parent cell-specific molecules, we hypothesized that macrophage exosomes (MФ-Exos) have a modulating effect on inflammation, osteogenesis, and angiogenesis. Hence, in this work, МФ-Exos were selected for the exploration of the regenerative potential of immune cell-derived exosomes in bone regenerative medicine. Biomaterials have been demonstrated to modulate the behaviors of macrophages to improve tissue regeneration. ${ }^{12}$ Biomaterials can significantly enhance the paracrine activity of cells and thus affect exosome secretion and biological functionality. Among them, bioceramics have been proven to be important regulators of the immunomodulation of macrophages via ion products, such as $\mathrm{SiO}_{4}{ }^{4-}, \mathrm{Ca}^{2+}, \mathrm{Mg}^{2+}$, etc. ${ }^{13}$ Bioceramic-stimulated macrophages displayed a marked change in the secretion of cytokines, chemokines, and proteases to regulate inflammation and subsequent osteogenesis/angiogenesis. Whether the paracrine pathway mediated by bioceramic-induced macrophage exosomes (BC-Exos) plays an important role in bioceramicmediated bone regeneration has not been fully elucidated. Therefore, this work investigated the regenerative potential of BC-Exos in cell-free tissue engineering and nanomedicine.

Herein, in this study, we designed a cell-free BC-Exo scaffold that exhibited a controlled porous structure and sustained exosome release for the regulation of immunomodulation, osteogenesis, and angiogenesis (Scheme 1). BC-Exo scaffolds were fabricated by extrusion-based 3D printing with bioink consisting of nanosized BC-Exos, sodium alginate (ALG), and hyaluronic acid (HA). Threedimensional printing resulted in the predefined porous structure of BC-Exo scaffolds due to the good printability of the ALG-HA hydrogel system. The release of BC-Exos improved the immunomodulatory, osteogenic and angiogenic ability of the scaffolds. The design of cell-free 3D printed scaffolds with bioceramic-induced exosomes may represent a direction for the development of tissue regeneration and repair biomaterials.

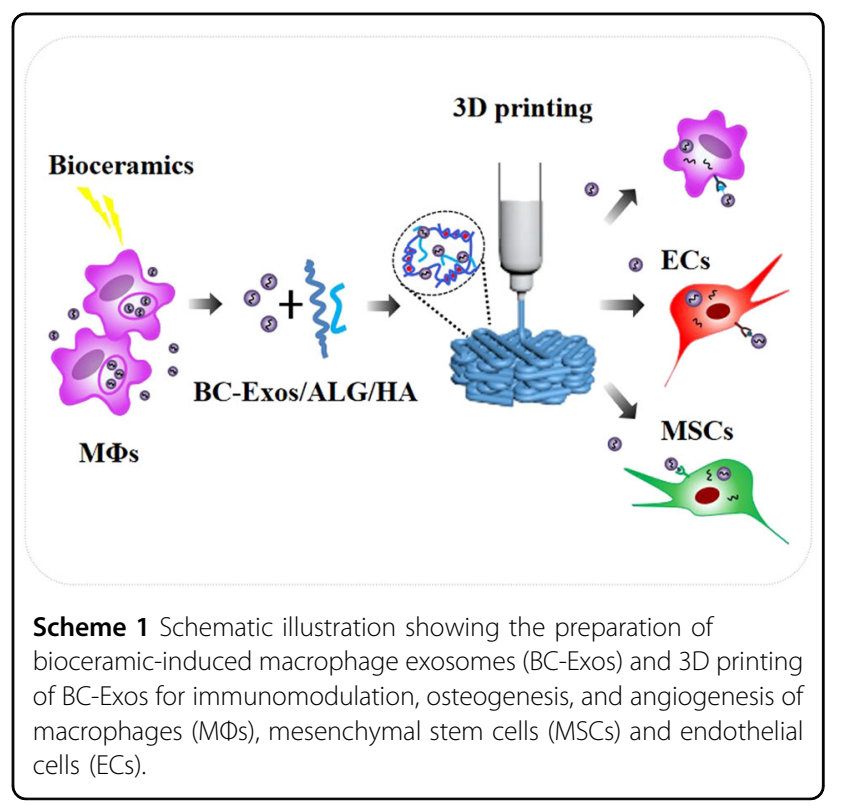




\section{Materials and methods Material preparation}

$\beta$-TCP was synthesized by the coprecipitation method. The phase composition of $\beta$-TCP powders was detected by X-ray diffraction (XRD, D8 Advance, Bruker AXS $\mathrm{GmbH}$, Germany). For the preparation of the $\beta$-TCP extract, $\beta$-TCP ceramic powder was added to Dulbecco's modified Eagle's medium (DMEM, Gibco, USA) with a mass volume ratio of $200 \mathrm{mg} / \mathrm{mL}$. After the mixture was shaken at $120 \mathrm{rpm} / \mathrm{min}$ and $37^{\circ} \mathrm{C}$ for $24 \mathrm{~h}$, it was centrifuged at $4000 \mathrm{rpm}$ for $10 \mathrm{~min}$. The supernatant was filtered through a $0.2 \mu \mathrm{m}$ filter membrane to obtain $200 \mathrm{mg} / \mathrm{mL} \beta$-TCP extract solution. The concentrations of $\mathrm{Ca}^{2+}$ and $\mathrm{PO}_{4}{ }^{3-}$ in the $\beta$-TCP extract were analyzed by inductively coupled plasma atomic emission spectroscopy (ICP-AES, Varian, USA).

\section{Macrophage activation with $\beta$-TCP extracts}

Macrophages (RAW 264.7) were cultured in DMEM complete medium (10\% fetal bovine serum (FBS, Gibco, USA), $100 \mu \mathrm{g} / \mathrm{mL}$ penicillin and $100 \mu \mathrm{g} / \mathrm{mL}$ streptomycin (Hyclone, USA)) at $37^{\circ} \mathrm{C}$ and $5 \% \mathrm{CO}_{2}$. The $\beta$-TCP extract was diluted to 25,50 , and $100 \mathrm{mg} / \mathrm{mL}$ and supplied with serum, penicillin, and streptomycin (named 25T, 50T, and $100 \mathrm{~T}$, respectively). Complete medium with no extract supplement was referred to as $0 \mathrm{~T}$. The proliferation of macrophages was analyzed by CCK- 8 assays at Days 1, 3, 5, and 7. A calcein-AM/propidium iodide (PI) double staining kit was used to observe both living and dead cells. For observation of cell morphology, MФs were stained with TRITC-phalloidin (Sigma-Aldrich, USA) and 4,6diamino-2-phenyl indole (DAPI, Sigma-Aldrich, USA). Gene expression was measured by real-time qPCR following a previous protocol. ${ }^{14}$ The primers were designed based on cDNA sequences from the NCBI Sequence Database and synthesized by Sangon Biotech (as shown in Table S1).

\section{Exosome purification and characterization}

Exosomes derived from the macrophage supernatant with or without $\beta$-TCP stimulation were prepared. Macrophages were gently washed in PBS and continuously cultured in a complete medium supplemented with $\beta$-TCP extracts and 10\% exosome-deprived FBS (SBI, USA) for 2 days and 4 days. Macrophage supernatant was centrifuged at $300 \times g$ for $10 \mathrm{~min}$ and $2000 \times g$ for $20 \mathrm{~min}$ to remove the cellular debris. Then, the supernatant was filtered through a $0.2 \mu \mathrm{m}$ sterilized filter to remove impurities, including particles larger than $0.2 \mu \mathrm{m}$. Then, exosomes were isolated from macrophage supernatant using Total Exosome Isolation Reagent (Invitrogen, USA) or polyethylene glycol (PEG)/ $\mathrm{NaCl}$ precipitation (16\% (w/ v) PEG6000, $1 \mathrm{M} \mathrm{NaCl}$ ). Exosomes were centrifuged at $10000 \times g$ for $60 \mathrm{~min}$, resuspended in PBS, and stored at $-80^{\circ} \mathrm{C}$ until use in subsequent experiments. For exosome identification, transmission electron microscopy (TEM, JEM-1400, Japan) was used to detect exosome morphology by staining with $3 \%(\mathrm{w} / \mathrm{v})$ phosphotungstic acid (pH 7.0). A NanoBrook ZetaPlus System (90Plus PALS, Brookhaven, USA) was used to measure the size distribution. The total protein concentration of exosomes was quantified using a Pierce ${ }^{\mathrm{TM}}$ BCA Protein Assay Kit (Thermo Fisher Scientific, USA), and the ExoELISAULTRA CD81 assay was used to quantitate CD81 ${ }^{+}$exosome abundance (SBI, USA). Western blotting analysis was performed to detect the exosomal markers CD9 (ab92726, Abcam, UK), CD81 (ab109201, Abcam, UK), and TSG101 (ab125011, Abcam, UK), with calnexin (ab22595, Abcam, UK), which is absent in exosomes, as a control.

\section{Exosome internalization}

Exosomes were first labeled with the green fluorescent cell linker PKH67 (Sigma-Aldrich, USA) according to the directions of PKH67 staining. BMSCs were incubated with PKH67-labeled exosomes for $12 \mathrm{~h}$ and then rinsed repeatedly and fixed in $4 \%$ paraformaldehyde for $30 \mathrm{~min}$. The cells were stained with TRITC-phalloidin and DAPI.

\section{RNA sequencing of exosomes}

The exosomal RNAs of E-0T-2D or E-25T-2D were isolated using RNAiso for small RNA (TaKaRa, Japan) $(n=3)$. Small RNA sequencing and analysis were conducted by Shanghai OE Biotech Co., Ltd. The known miRNAs were identified by alignment against the miRBase v.21 database (http://www.mirbase.org/), and their expression patterns in different samples were analyzed. Differentially expressed miRNAs were identified with the threshold of $p<0.05$, which was calculated with the differential expression analysis (DEG) algorithm in the $R$ package for experiments with biological replicates. The targets of differentially expressed miRNAs were predicted by using miRanda software in animals with the parameters $S \geq 150, \Delta \mathrm{G} \leq-30 \mathrm{kcal} / \mathrm{mol}$, and strict $5^{\prime}$ seed pairing. Gene Ontology (GO) enrichment and Kyoto Encyclopedia of Genes and Genomes (KEGG) pathway enrichment analyses of differentially expressed miRNAtarget-mRNAs were performed using $R$ based on the hypergeometric distribution. Sequencing data supporting these studies can be found in the Sequence Read Archive database under accession number SRR13781710.

\section{Effects of MФ-Exos on angiogenic activity of HUVECs}

МФ-Exos were diluted to 10,25 , and $40 \mu \mathrm{g} / \mathrm{mL}$, and the proliferation of human umbilical vein endothelial cells (HUVECs) cultured with different exosomes was measured by CCK-8 assays at Days 1, 3, 5, and 7. For the wound healing assay, ECM medium (ScienCell, USA) 
containing $1 \%$ serum with different $M \Phi$-Exos was added to each well. The migration rate (\%) of HUVECs was calculated after $14 \mathrm{~h}$. HUVECs were fixed and stained with $0.2 \%$ crystal violet. The Transwell assay was performed by using Transwell inserts with $8 \mu \mathrm{m}$ pore size. After removal of the nonmigrating cells, HUVECs stained with $0.2 \%$ crystal violet on the outside of the filters were photographed. Afterward, the crystal violet was decolorized by adding $300 \mu \mathrm{L}$ of $33 \%$ acetic acid, and the absorbance value was measured at $570 \mathrm{~nm}$. The in vitro angiogenesis assay was conducted using the Matrigel ${ }^{\mathrm{TM}}$ basement membrane matrix (Corning, USA). HUVECs were resuspended in a complete medium supplemented with $M \Phi$-Exos and then cultured on solidified Matrigel. After $6 \mathrm{~h}$, the cells were stained with calcein-AM and photographed. The numbers of tubes formed and branch points were calculated by Image-Pro Plus software. The gene expression of HUVECs at $24 \mathrm{~h}$ and Day 3 was measured by real-time qPCR. The primer sequences are listed in Table S2.

\section{Effects of MФ-Exos on immunomodulation of BMSCs}

Mouse bone marrow mesenchymal stem cells (BMSCs) cultured with MФ-Exos at $10 \mu \mathrm{g} / \mathrm{mL}$ for 3 days were lysed for RNA extraction and further detected by real-time qPCR. The primer sequences are listed in Table S1.

\section{Effects of MФ-conditioned media on osteogenic differentiation of BMSCs}

MФs were cultured in $\beta$-TCP extracts (10\% exosomedeprived FBS) supplemented with $10 \mu \mathrm{M}$ GW4869 to inhibit BC-Exo production. After 1 day, the MФs were cocultured in $\beta$-TCP extracts for another 24h. Media from the MФs with or without GW4869 (Sigma-Aldrich, USA) were collected, centrifuged at $1500 \mathrm{rpm}$, and supplemented with BMSC culture medium at a ratio of $1: 1$ to obtain MФ-conditioned media. Gene expression of the BMSCs cultured in MФ-conditioned media was detected by real-time qPCR at Day 3.

\section{Three-dimensional bioprinting and characterization of scaffolds}

Bioink for 3D printing was prepared with $10 \%(\mathrm{w} / \mathrm{v})$ alginate (ALG, Acros, China) and 5\% (w/v) hyaluronic acid (HA, Bloomage Biotech, China, 200-400 kDa) dissolved in PBS. Three-dimensional scaffolds were printed layer by layer with a scaffold 3.0 printer and crosslinked with $2 \% \mathrm{CaCl}_{2}$ solution. For $3 \mathrm{D}$ printing of scaffolds integrated with BC-Exos, ALG/HA bioink containing 100, 200, and $400 \mu \mathrm{g} / \mathrm{mL}$ BC-Exos was also prepared. PKH67labeled BC-Exos were mixed well with ALG and HA powders in PBS. The printed scaffolds were observed using a confocal laser scanning microscope (SP8 LIGHTNING, Leica, Germany). The rheological properties of the hydrogel scaffolds were studied by using a $25 \mathrm{~mm}$ parallel-plate rheometer (Physica MCR 301, Anton Paar, Austria). The elastic moduli $\left(G^{\prime}\right)$ and viscous moduli $\left(G^{\prime \prime}\right)$ were measured in two modes. The frequency sweep was performed from 0.01 to $80 \mathrm{~Hz}$ frequency at $1 \%$ strain, and the strain sweep was performed from 0.01 to $1000 \%$ at $1 \mathrm{~Hz}$. For analysis of the degradation of the hydrogels, 3D scaffolds were submerged in $0.9 \% \mathrm{NaCl}$ solution for 4 weeks. The mass of the scaffolds was weighed at designed time points $\left(W_{n}\right)$ after vacuum freeze-drying. The degradation ratio was determined as $\left(W_{0}-W_{\mathrm{n}}\right) / W_{0} \times 100$, where $W_{0}$ is the initial mass of the scaffold $(n=3)$. The release of exosomes was detected by a Pierce $^{\mathrm{TM}}$ BCA Protein Assay Kit. BC-Exo scaffolds were submerged in cell basal medium, and the retention ratio was determined as $\left(M_{0}-M_{\mathrm{n}}\right) / M_{0} \times 100$, where $M_{0}$ represents the initial loading amount of the BC-Exos in scaffolds and $M_{\mathrm{n}}$ represents the cumulative released amount $(n=3)$.

\section{Immunomodulation of MФs on BC-Exo scaffolds}

After culture for 1 day, living/dead cells on scaffolds were stained with calcein-AM/PI. Real-time PCR was applied to detect the gene expression of MФs on the scaffolds at Day 3. The primer sequences are listed in Table S3. Supernatants from the BC-Exo scaffold-stimulating MФs were collected, centrifuged at $1500 \mathrm{rpm}$, and supplemented with BMSC or ECM culture medium at a ratio of 1:1 to obtain $М Ф$-conditioned media. A woundhealing assay was performed to explore the effects of the BC-Exo scaffold-stimulated MФs on HUVEC migration. After incubation for $17 \mathrm{~h}$ in $\mathrm{M} \Phi$-conditioned media, the migration rate of HUVECs was analyzed with Image-Pro Plus Software. Transwell assays were performed to detect the effect of BC-Exo scaffold-stimulated MФs on BMSC chemotaxis.

\section{Osteogenesis of BMSCs on BC-Exo scaffolds}

After culture for 1 day and 14 days, living/dead cells on scaffolds were stained with calcein-AM/PI, and cell adhesion and spread were observed by immunofluorescence staining with TRITC-phalloidin and DAPI. Cell proliferative activity was detected by the CCK- 8 method. Real-time qPCR was applied to detect the expression of genes involved in immunosuppression at Day 3 and osteoblastic differentiation at Day 14. The primer sequences are listed in Table S1.

\section{Angiogenesis of HUVECs on BC-Exo scaffolds}

HUVECs were seeded on 3D scaffolds with different types of $M \Phi-E x o s$. Cell proliferation activity was detected by the CCK-8 method, and calcein-AM/PI was used to label the living/dead cells on the scaffolds at Day 1. Cell adhesion at one day was observed by staining with 

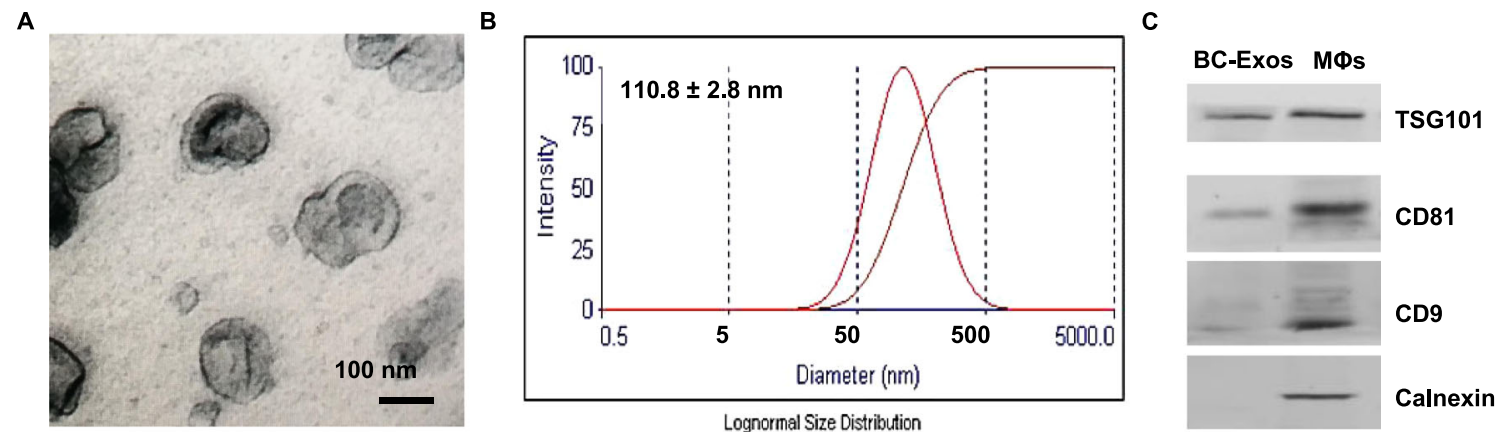

Fig. 1 Characterization of bioceramic-induced macrophage exosomes (BC-Exos). A Morphology of BC-Exos visualized under TEM. B Particle size distribution. C Characteristic surface markers of CD9, CD81, and TSG101 by western blotting, and calnexin was considered cell contamination. BC-ExOS had a cup-shaped morphology with a size of approximately $110 \mathrm{~nm}$, and they were purified with characteristic exosomal surface marker proteins, including CD9, CD81, and TSG101.

TRITC-phalloidin. The expression of angiogenesis-related genes was detected at Day 14. The primer sequences are listed in Table S2.

\section{Statistical analysis}

All data are presented as the mean \pm standard deviation (SD). The statistical analysis of two groups was performed in SPSS Statistics 17.0 using one-way or two-way ANOVA with Tukey's post hoc multiple comparisons test. For all analyses, a $p$-value $<0.05$ was considered to indicate statistical significance. The dependent variables were normally distributed for the groups formed by the combination of the levels of sample and culture time as assessed by the Shapiro-Wilk test. There was homogeneity of variance between groups as assessed by Levene's test for equality of error variances.

\section{Results and discussion}

\section{Characterization of bioceramic-induced macrophage} exosomes (BC-Exos)

Here, $\beta$-tricalcium phosphate $(\beta$-TCP), a remarkable bone-repairing bioceramic in clinical applications, was chosen as the model material for the induction of $\mathrm{BC}$ Exos. XRD analysis of the $\beta$-TCP powders is shown in Fig. S1. To identify the purified BC-Exos, we performed TEM, particle size analysis, and western blotting (Fig. 1). TEM images showed that the BC-Exos had a cup-shaped morphology with a nanosize of approximately $100 \mathrm{~nm}$ (Fig. 1A). The particle size measurement revealed that the size of the BC-Exos was $110.8 \pm 2.8 \mathrm{~nm}$ (Fig. 1B). CD9, CD81, and TSG101 are pan-exosome markers, and calnexin is a common cell contaminant. To determine whether the BC-Exos expressed exosomal markers or contained contaminants, we performed western blotting analysis. The results showed that the BC-Exos were positive for characteristic exosomal surface marker proteins, including CD9, CD81, and TSG101. Compared with
$\mathrm{M \Phi s}$, the BC-Exos were devoid of the endoplasmic reticulum protein calnexin (Fig. 1C). This finding indicates that the purified $\mathrm{BC}$-Exos were successfully prepared in this work.

\section{Three-dimensional printing of BC-Exos scaffolds}

Bioink composed of sodium alginate (ALG) and hyaluronic acid (HA) was further applied for 3D printing of nanosized BC-Exos. Printability of ALG-HA bioink was first verified. Following crosslinking by $\mathrm{Ca}^{2+}$, resilient selfsupporting ALG-HA scaffolds were obtained, as shown in Fig. S2. The ALG-HA bioink showed good printability with different angles, diameters, and heights (Fig. S2A $-D)$. The scaffolds were elastic and strong enough to withstand various forms of deformation (Fig. S2E, F) and supported cell adhesion and growth (Fig. S2G). With integrated BC-Exos, the printability of bioink was less affected. Figure $2 \mathrm{~A}-\mathrm{E}$ presents the $3 \mathrm{D}$ printing of the $\mathrm{BC}-$ Exos with different angles, diameters, root distances, heights, and shapes. The BC-Exo scaffolds can bear twisting and compression (Fig. 2F, G). The rheological properties of the scaffolds with and without BC-Exos (referred to as $\mathrm{S}+\mathrm{BC}$-Exos and $\mathrm{S}$, respectively) are shown in Figs. 2H, I. Both the storage modulus $\left(G^{\prime}\right)$ and loss modulus $\left(G^{\prime \prime}\right)$ displayed an increasing trend with increasing frequency at $1 \%$ constant strain, and $G^{\prime}$ was always higher than $G^{\prime \prime}$, which indicates the solid-like and elastic characteristics of the hydrogels. Comparing the influence of BC-Exos on the modulus of the gel, we found that the $G^{\prime}$ and $G^{\prime \prime}$ of the BC-Exo scaffolds showed an obvious increase. $\mathrm{BC}$-Exos may enhance the density of the hydrogel network by serving as nanoparticle fillers and thus enhance the scaffold modulus. At a constant frequency $(1 \mathrm{~Hz}), G^{\prime}$ decreased gradually with increasing strain amplitude due to the disruption of hydrogen bonding crosslinking. This phenomenon is a typical rheological behavior of a physical crosslinking network. 


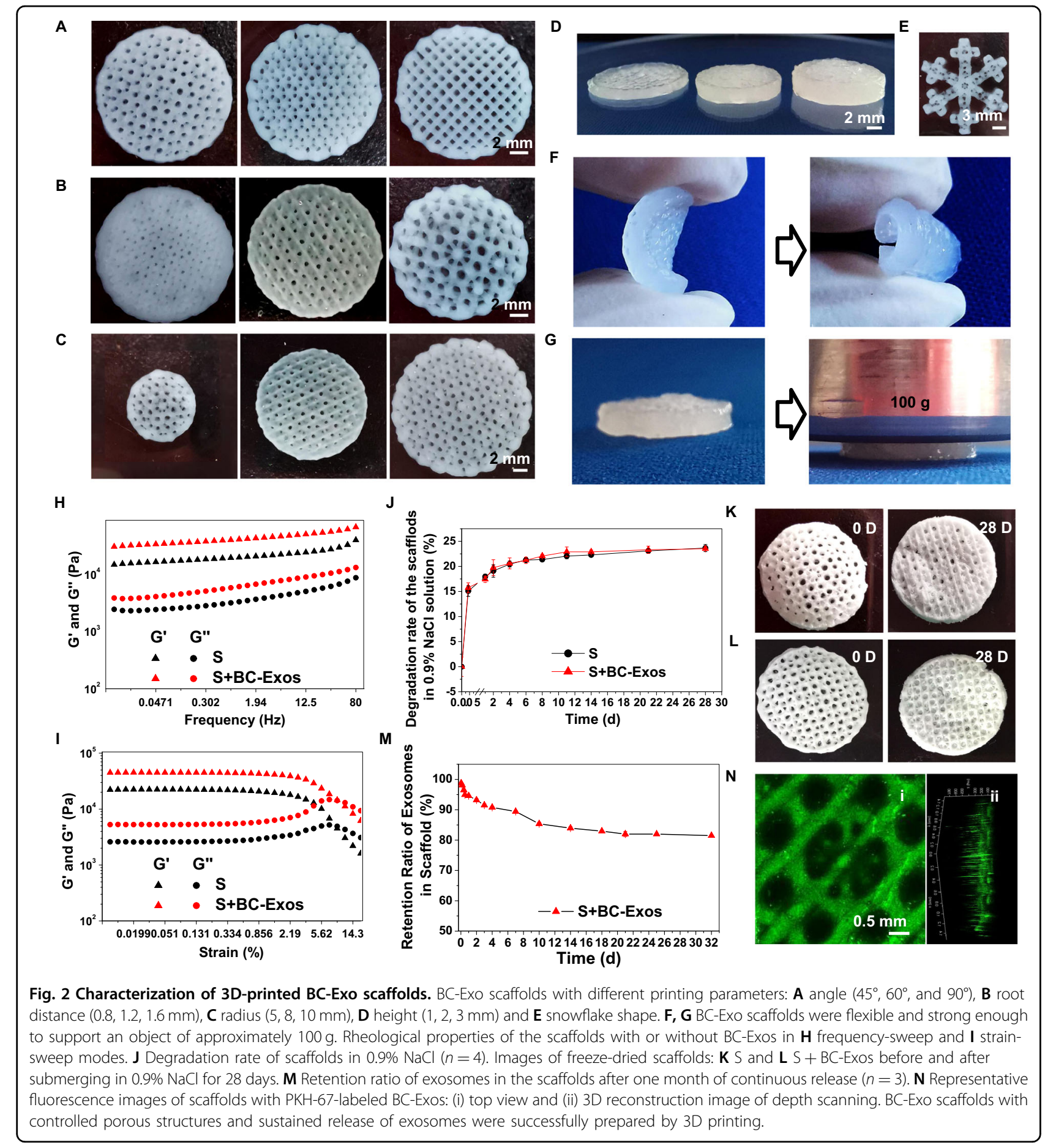

Moreover, $G^{\prime \prime}$ exhibited a gradual increment at the beginning, which was caused by the continuous destruction and reorganization of crosslinking, and then decreased after reaching the peak value. Figure 2J shows that the mass loss of the BC-Exo scaffolds in $0.9 \% \mathrm{NaCl}$ solution for 4 weeks was approximately $24 \%$. The scaffolds still maintained the 3D printed morphology (Fig. 2K, $\mathrm{L})$. The release profile of BC-Exos was investigated in a cell basal medium. As shown in Fig. 2M, there was a sudden release of $\mathrm{BC}$-Exos within one day, followed by a long-term sustained release. The retention rate of $\mathrm{BC}$ Exos in scaffolds was approximately $80 \%$ after one month. This result indicated that 3D printing of BC-Exos can achieve the long-term release of $\mathrm{BC}$-Exos, which is beneficial for sustained tissue regeneration. The fluorescence images in Fig. $2 \mathrm{~N}$ show the uniform distribution of 
PKH67-labeled BC-Exos in scaffolds. BC-Exo scaffolds with controlled porous structures and sustained release of exosomes were successfully prepared by 3D printing.

\section{Three-dimensional printing of BC-Exos regulated the immunomodulation of MФs}

$M \Phi$-Exos have been reported to accelerate wound healing through their anti-inflammatory effects in a diabetic rat model. ${ }^{15}$ To investigate the roles of BC-Exos in the regulation of the inflammatory response, we isolated BC-Exos induced by 0,25 , and $100 \mathrm{mg} / \mathrm{mL} \quad \beta$-TCP extracts for 2 days; these samples were referred to as E0T-2D, E-25T-2D, and E-100T-2D, respectively. Given the greater efficiency of promoting BMSC proliferation and osteogenic differentiation, a BC-Exo concentration of $200 \mu \mathrm{g} / \mathrm{mL}$ in scaffolds was chosen (Figs. S3 and S4). Scaffolds integrated with E-0T-2D, E-25T-2D and E100T-2D were named SE-0T-2D, SE-25T-2D and SE100T-2D, respectively. BC-Exos promoted the adhesion of МФs on scaffolds, and the cells maintained good activity (Fig. S5A). The results in Fig. 3A show that MФ-Exo scaffolds enhanced the gene expression of interleukin (IL) 6 in MФs but decreased the IL10 and transforming growth facter- $\beta 1$ (TGF- $\beta 1$ ) levels. Compared with those in the other groups, the MФs in the SE-25T-2D group obviously downregulated the expression of the proinflammatory genes inducible nitric oxide synthase 2 (iNOS2) and tumor necrosis factor- $\alpha$ (TNF- $\alpha$ ) and upregulated the expression of the anti-inflammatory genes arginase 1 (Arg1) and IL1ra, indicating a stronger M2 phenotype. M2-type macrophages have been studied extensively with respect to their secretion of antiinflammatory cytokines and ability to modulate tissue healing and regeneration. ${ }^{12}$ In the early stage of the response to injury, macrophages can also release cytokines and chemokines to recruit tissue repair cells, such as MSCs and endothelial cells, to the injured site. ${ }^{16}$ As shown in Fig. 3B, higher expression of vascular endothelial growth factor A (VEGFA) and stromal cell-derived factor-1 (SDF-1 $\alpha)$ was detected in the MФs on MФ-Exo scaffolds. In addition, the MФs in the SE-25T-2D group showed significantly higher expression of monocyte chemotactic protein-1 (MCP-1). VEGF can not only promote the mobilization and proliferation of endothelial progenitor cells but also has a chemotactic effect on endothelial progenitor cells, which can promote new vessel formation. SDF- $1 \alpha$ mediates the chemotaxis and migration of many cell types and plays an important role in stem cell homing. MCP-1 is known to contribute to monocyte/macrophage infiltration to the injured site. However, during angiogenesis, MCP-1 was shown to induce the migration of vascular endothelial cells. ${ }^{17} \mathrm{~A}$ wound-healing assay (Fig. 3C, D) revealed that conditioned media $(\mathrm{CM})$ from the $\mathrm{BC}$-Exo scaffold-stimulated
MФs significantly promoted the migration of HUVECs through paracrine effects. Moreover, the HUVECs in the CM-SE-25T-2D group showed the best migratory ability, which may be related to the highest expression of MCP-1 in MФs. Transwell assays of BMSCs suggested that the BC-Exo scaffold-stimulated MФs had a chemotactic effect on BMSCs (Fig. S5B). The data indicate that 3D printing of $\mathrm{BC}$-Exos can regulate both the inflammatory response and the recruitment of tissue repair cells mediated by macrophages, enhancing tissue repair.

\section{Three-dimensional printing of BC-Exos enhanced the osteogenesis of BMSCs}

Further exploration of the osteogenesis of 3D printed BC-Exos is shown in Fig. 4. Compared to that of the pure scaffolds, the long-term release of MФ-Exos significantly improved BMSC adhesion, proliferation, and the expression of genes involved in osteoblastic differentiation, such as alkaline phosphatase (ALP), type I collagen (COL I), osteopontin (OPN), TGF- $\beta 1$, bone morphogenetic protein 2 (BMP2) and VEGFA (Fig. 4A-D). Although the initial adhesion amount of BMSCs on scaffolds was lower owing to the hydrophilicity of hydrogels, many BMSCs with good cytoactivity and spreading were observed on the MФ-Exo scaffolds at Day 14. MФ-Exos may promote the expression of genes related to BMSC proliferation and adhesion. Exosomes derived from primary tumors have been found to facilitate metastasis by regulating the proliferation and adhesion of circulating tumor cells. ${ }^{18}$ Comparing the effect of $\beta$-TCP stimulation on BC-Exo functionality, we demonstrated that BMSC proliferation and osteogenic differentiation were significantly increased in the SE-25T2D group compared to the SE-0T-2D and SE-100T-2D groups. Higher expression of COL I, bone sialoprotein (BSP), OPN, TGF- $\beta 1$, VEGFA, and hypoxia-inducible factor-1 $\alpha$ (HIF-1 $\alpha)$ was detected in the SE-25T-2Dmodulated BMSCs. The BMP2/Smad pathway can trigger the osteogenesis of BMSCs by inducing the transcription of the target genes runt-related transcription factor 2 (Runx2) and osterix. Real-time qPCR detection indicated that the expression of bone morphogenetic protein receptor, type IA (BMPR1A), Smad1, and Samd5 was significantly upregulated in the SE-25T-2D group (Fig. S6). The improved osteogenic differentiation of BMSCs in the SE-25T-2D group was likely associated with a BMP2related mechanism. In addition to their multilineage differentiation ability, BMSCs are also known for their ability to restrain inflammation. Several mediators, such as prostaglandin E2 (PGE2), tumor necrosis factor-alpha stimulated gene-6 (TSG-6), galectin-1 (GAL-1), TGF- $\beta 1$, and IL- 6 , are produced by BMSCs upon inflammatory stimulation. BMSCs in the SE-25T-2D group significantly upregulated the expression of GAL-1, TSG-6, and IL6 (Fig. 4E). GAL-1 was proven to inhibit T-cell proliferation, 

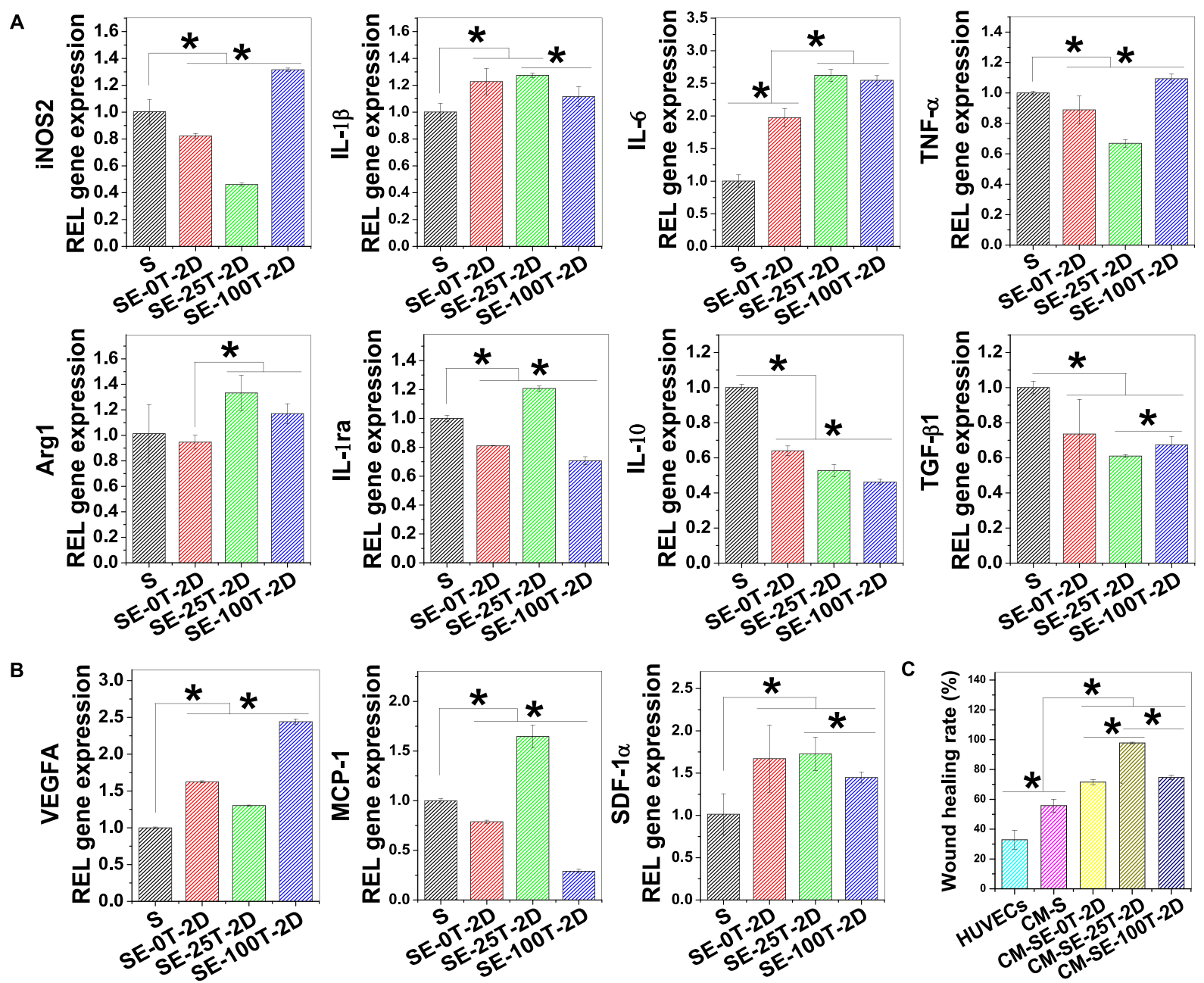

D
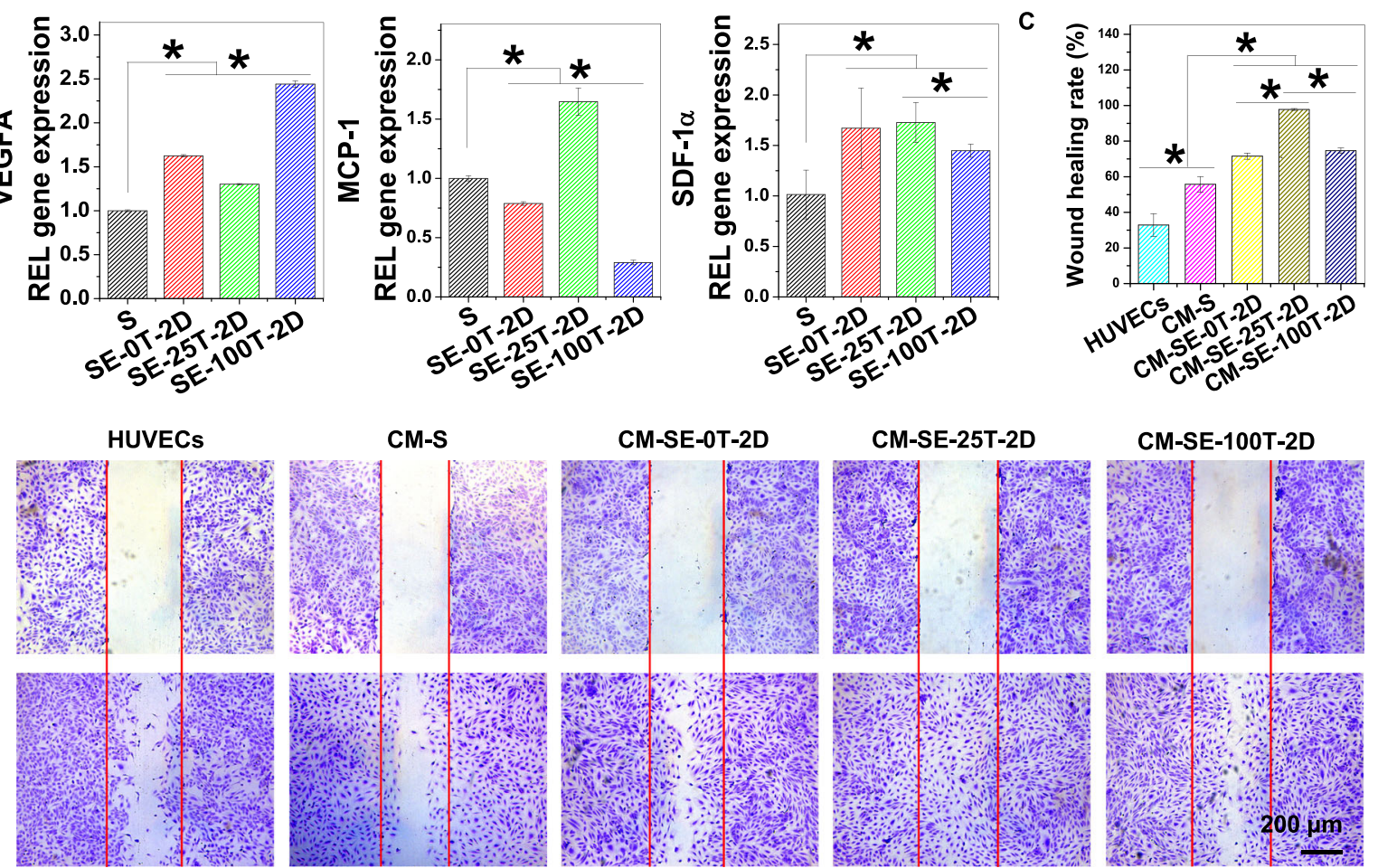

Fig. 3 Effects of 3D printing of BC-Exos on the immunomodulation of macrophages seeded on scaffolds. A Macrophage polarization at Day 3 $\left(n=4,{ }^{*} p<0.05\right)$. B Expression of migration-related genes in macrophages seeded on scaffolds at Day 3: VEGFA, MCP-1 and SDF-1a $\left(n=4,{ }^{*} p<0.05\right)$. C Effects of conditioned media from BC-Exo scaffold-stimulated macrophages on HUVEC migration $\left(n=5,{ }^{*} p<0.05\right)$. D Representative images of the wound healing assay of HUVECs. Three-dimensional printing of BC-Exos regulated the inflammatory response of macrophages and promoted the macrophage-mediated migration of endothelial cells. 


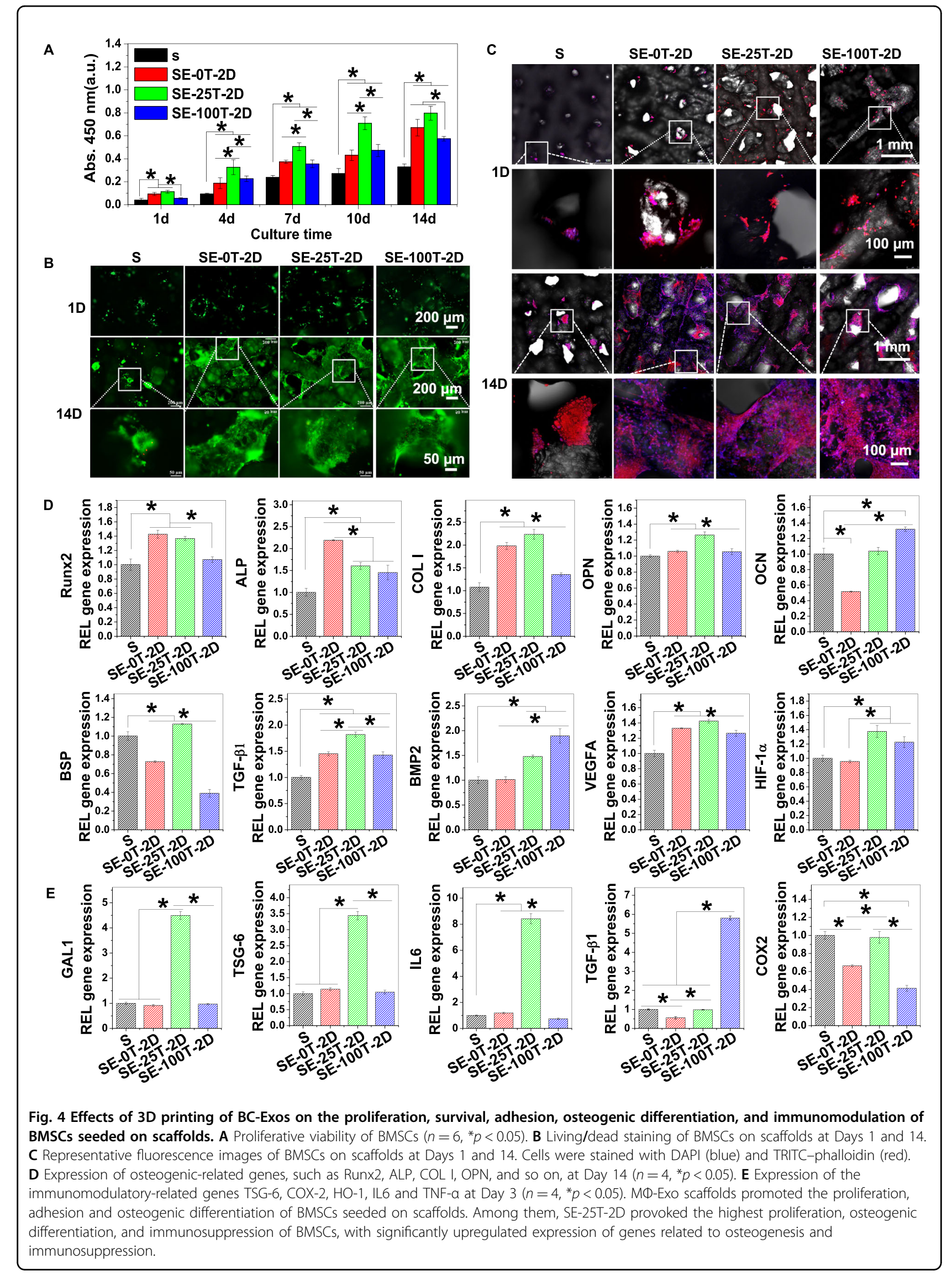




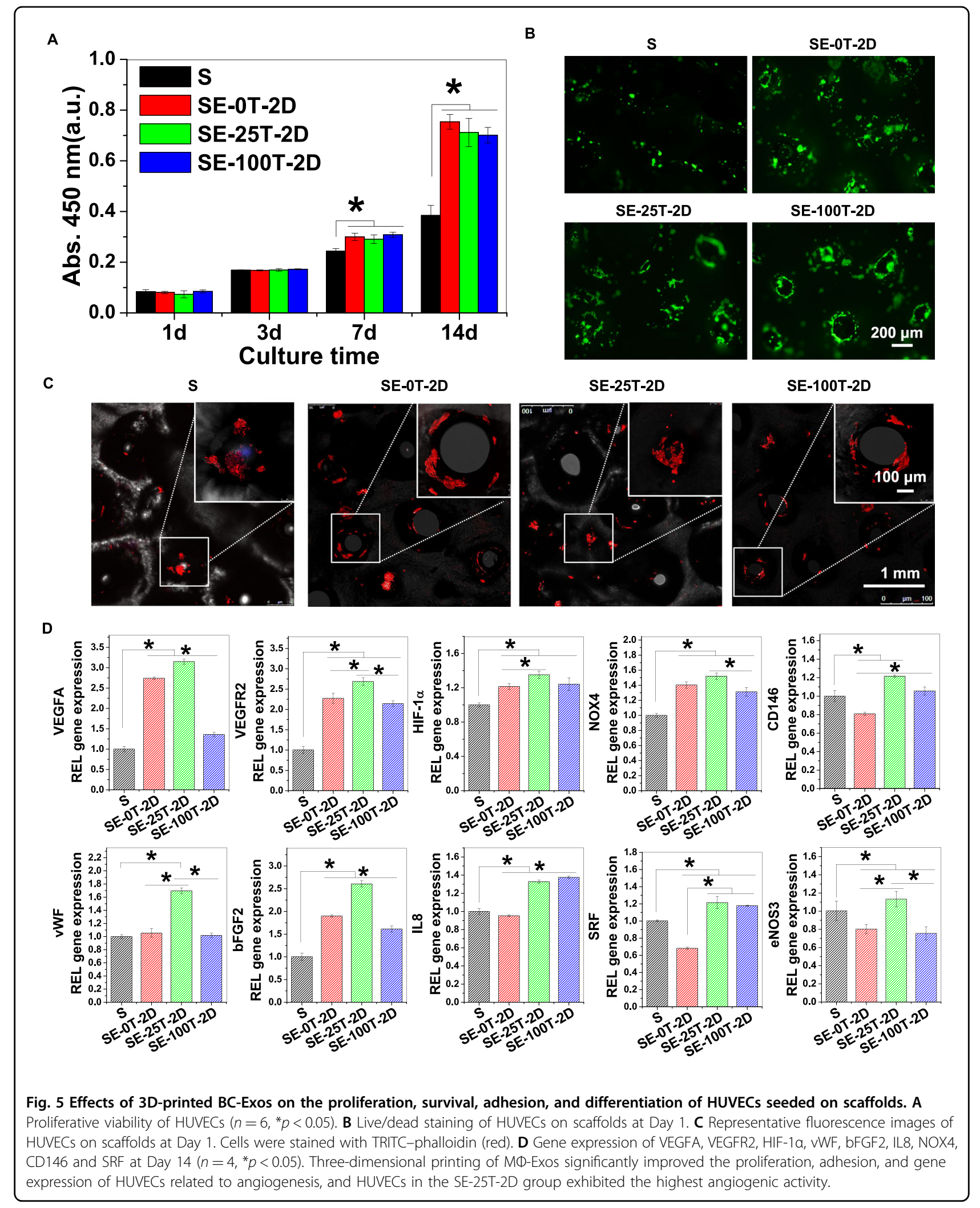


and MSC-mediated T-cell suppression was inhibited by GAL-1-specific siRNAs. ${ }^{19}$ TSG6 is an anti-inflammatory protein produced by MSCs in response to tissue injury that can induce a macrophage phenotypic switch to the M2 type. ${ }^{20}$ Although IL6 is best known as a kind of proinflammatory cytokine, there is a parallel body of research that has proven the role of IL6 in immunosuppression and bone formation. ${ }^{21,22}$ MSCs inhibited the differentiation of dendritic cells and local inflammation in an experimental mouse arthritis model through an IL6dependent mechanism. Further confirmation that the BCExos elicited an immunosuppressive effect on BMSCs is shown in Fig. S7. BMSCs were directly treated with $10 \mu \mathrm{g} /$ $\mathrm{mL} \mathrm{BC}$-Exos for 3 days. The results showed that the expression of TSG-6 and cyclooxygenase-2 (COX-2) was distinctly enhanced. COX-2 is a specific oxidase in prostaglandin E2 (PGE2) production. PGE2 is another immunosuppressive molecule produced by inflammatory stimulus-induced BMSCs. Research on the immunomodulatory effects of MSCs has revealed that MSCs are not spontaneously immunosuppressive, and priming by inflammatory cytokines is essential for MSC-mediated immunosuppression. ${ }^{23}$ This finding suggests that the immunosuppressive effect of BMSCs might be initiated by inflammatory factors transferred from BC-Exos. The fluorescence images in Fig. S8 show that PKH67-labeled BC-Exos (green) were located around the nucleus of BMSCs (blue), which is indicative of phagocytosis and offers a biological basis for the effect of BC-Exos on BMSCs. Based on the above results, we hypothesized that BC-Exos play a key role in osteogenesis through two different mechanisms: one is to promote the directional osteogenic differentiation of BMSCs to repair the damaged tissue; the other is to induce the secretion of immunosuppressive factors in BMSCs to inhibit the progress of local inflammation to promote tissue repair and regeneration.

\section{Three-dimensional printing of BC-Exos improved the angiogenesis of HUVECs}

Angiogenesis is a vital process for bone healing and remodeling. To investigate the effect of BC-Exos on angiogenesis, we assessed the angiogenic activity of HUVECs on BC-Exo scaffolds. Figure 5 shows that 3D printing of MФ-Exos significantly improved HUVEC proliferation, adhesion, and gene expression of VEGFA, vascular endothelial growth factor receptor 2 (VEGFR2), HIF-1 $\alpha$, basic fibroblast growth factor 2 (bFGF2) and $\mathrm{NADPH}$ oxidase 4 (NOX4). Moreover, BC-Exos enhanced the promotion of MФ-Exos on the expression of HIF-1 $\alpha$, IL8, CD146, and serum response factor (SRF). HUVECs in the SE-25T-2D group exhibited the highest angiogenic activity. Growth factors, such as VEGFA, von Willebrand factor (vWF), HIF-1 $\alpha$, bFGF2, and IL8, can regulate endothelial cell proliferation, migration, and tube formation, playing critical roles in angiogenesis. NOX4 can promote endothelial cell proliferation and tube formation through stabilization of HIF- $1 \alpha$ and induction of VEGF expression, while CD146 is a member of the cell adhesion molecules expressed on endothelial cells, which is required for cell migration and tube formation. SRF, an evolutionarily conserved transcription factor, was reported to selectively regulate migratory tip cell behavior during sprouting angiogenesis. ${ }^{24}$ Increased expression of these angiogenesis-related genes indicated the positive effect of BC-Exos on angiogenesis. To further confirm the role of BC-Exos in angiogenesis, we directly cultured HUVECs with MФ-Exos. HUVECs showed increased proliferation and upregulated expression of VEGFR2, HIF- $1 \alpha$, and bFGF2 after treatment with $10 \mu \mathrm{g} / \mathrm{mL} \mathrm{BC-}$ Exos for $24 \mathrm{~h}$ (Fig. S9). Further comparison of the influence of BC-Exos on HUVECs showed that the relative expression of angiogenesis-related genes in the E-25T-2D group at 3 days was obviously higher than that in the blank group (Fig. S10A). BC-Exos improved MФ-Exomediated HUVEC migration and tube formation (Fig. S10B-H). The data indicate that BC-Exos may be potential angiogenic agonists for angiogenesis.

\section{$\beta$-TCP stimulation altered the MФ-exosomal miRNA profiles}

Exosomes contain a variety of RNAs, proteins, and lipids. Intensive studies have focused on exosomal contents, including proteins and RNAs, and have attempted to identify their underlying mechanisms in tissue healing applications. It is believed that miRNAs are one of the main functional components of exosomes in regulating the balance of bone metabolism. ${ }^{25}$ To explore the reason for the biofunctional differences of $М \Phi$-Exos, we further studied the effect of $\beta$-TCP extracts on M $\Phi$-exosomal miRNA cargos. Global small RNA sequencing and difference analysis of E-0T-2D and E-25T-2D were performed. Over 1000 miRNAs were detected in the MФExos (SRA database SRR13781710). After removal of the weakly differentially expressed miRNAs, 78 miRNAs showed upregulated expression and 74 miRNAs showed downregulated expression in E-25T-2D compared to E0T-2D ( $\geq 2.0$-fold, $p<0.05)$, as shown in Fig. $6 \mathrm{~A}$ and Table S3. GO analysis of the target mRNAs of the top 30 differentially expressed miRNAs revealed that these target mRNAs could regulate biological processes, such as DNA transcription and cell differentiation, adjust cellular components in the cytoplasm, membrane, and nucleus, and influence the molecular functions of protein binding, metal ion binding and DNA binding (Fig. S11). KEGG signaling pathway enrichment analysis showed that the target mRNAs of the top 20 differentially expressed miRNAs exhibited a significant enrichment of signaling 
A

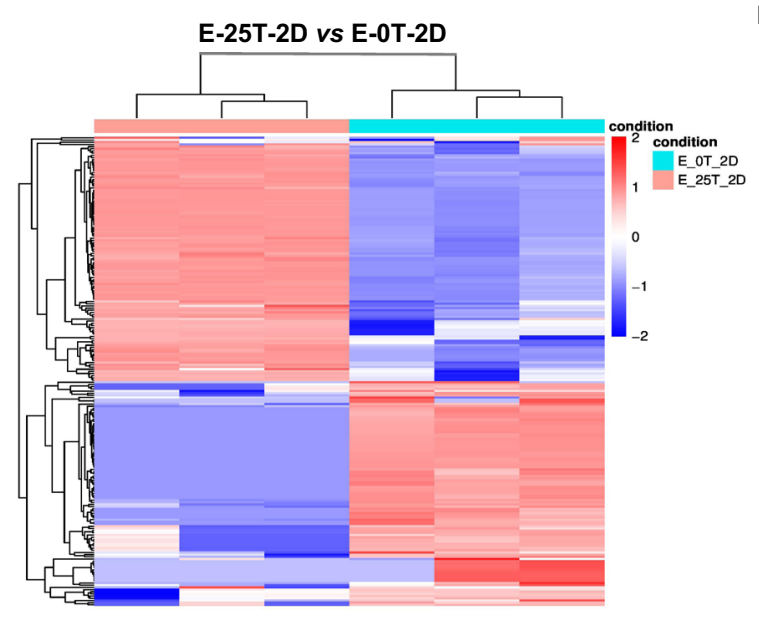

C

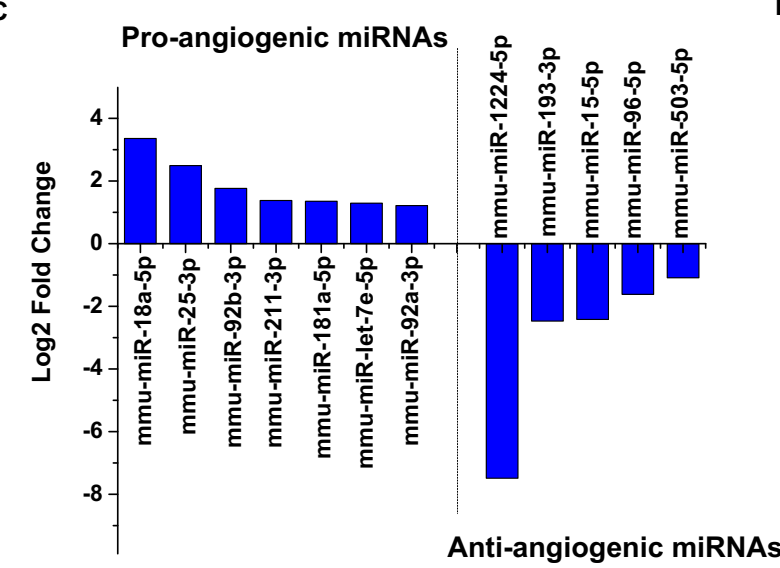

B

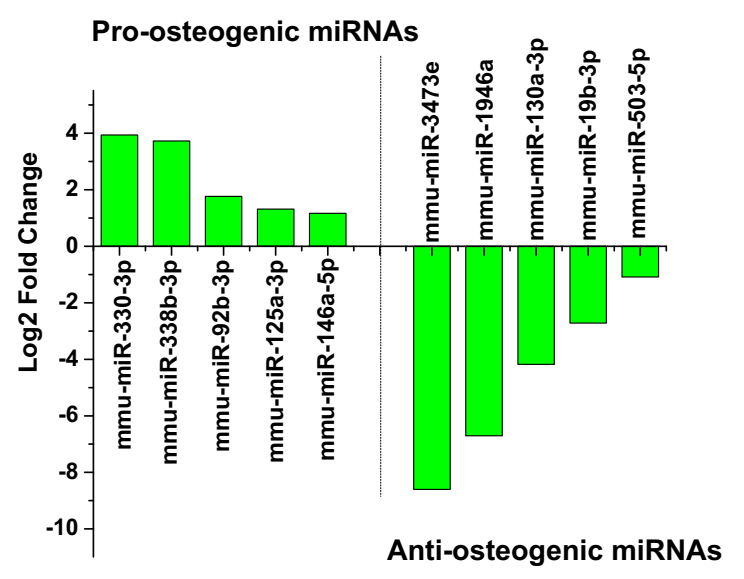
Anti-inflammatory miRNAs

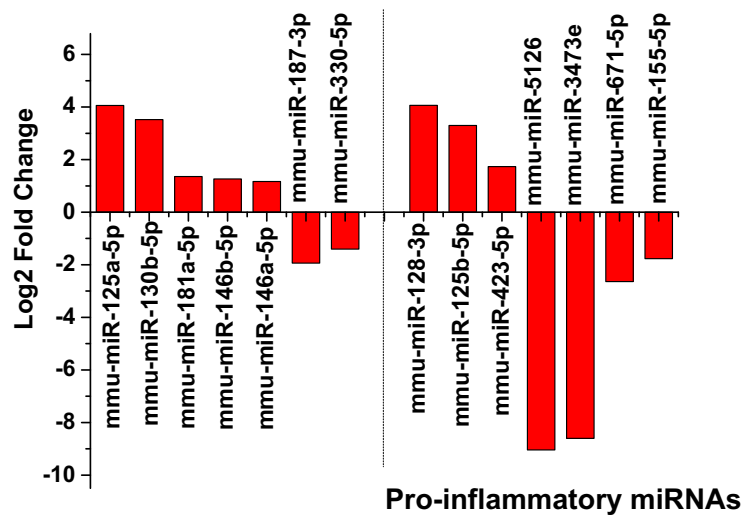

Fig. $6 \beta$-TCP stimulation altered the miRNA profiles of BC-Exos. A Heat map of the miRNAs with upregulated and downregulated expression with a cutoff value $\geq 2$-fold difference between E-25T-2D and E-0T-2D $(p<0.05)$. Representative miRNAs with upregulated and downregulated expression involved in $\mathbf{B}$ osteogenesis, $\mathbf{C}$ angiogenesis, and $\mathbf{D}$ immunomodulation in E-25T-2D compared to E-0T-2D. Pro-osteogenic miRNAs, such as mmu-miR-330-3p, mmu-miR-338-3p and mmu-miR-146a-5p; proangiogenic miRNAs, such as mmu-miR-18a-5p, mmu-miR-25a-3p and mmu-miR92b-3p; and anti-inflammatory miRNAs, such as mmu-miR-125a-5p, mmu-miR-130b-5p and mmu-miR-146b-5p, showed significantly upregulated expression in E-25T-2D compared to E-0T-2D. Instead, antiosteogenic miRNAs, including mmu-miR-3473e, mmu-miR-1946a and mmu-miR-19b-3p, antiangiogenic miRNAs, such as mmu-miR-1224-5p, mmu-miR-193a-3p, and mmu-miR-15b-5p, and proinflammatory miRNAs, such as mmu-miR5126, mmu-miR-3473e, and mmu-miR-155-5p, showed downregulated expression in E-25T-2D. Macrophages responded to $\beta-T C P$ stimulation by releasing exosomes containing highly specific RNA cargos, and the alterations of miRNAs in exosomes may play important roles in regulating immunomodulation, osteogenesis, and angiogenesis.

pathways such as the MAPK signaling pathway, calcium signaling pathway, Wnt signaling pathway, adhesion junction, and signaling pathways regulating pluripotency of stem cells (Fig. S12). Further analysis of the miRNA profiles revealed that pro-osteogenic miRNAs, such as mmu-miR-330-3p, mmu-miR-338-3p, mmu-miR-125a$3 p$ and mmu-miR-146a-5p, had upregulated expression in E-25T-2D, while some antiosteogenic miRNAs, including mmu-miR-3473e, mmu-miR-1946a, mmu-miR130a-3p and mmu-miR-19b-3p, had downregulated expression compared to those in E-0T-2D (Fig. 6B). Moreover, proangiogenic miRNAs, such as mmu-miR18a-5p, mmu-miR-25a-3p and mmu-miR-92b-3p, and antiangiogenic miRNAs, such as mmu-miR-1224-5p, mmu-miR-193a-3p and mmu-miR-15b-5p, showed significantly upregulated and downregulated expression in E-25T-2D, respectively (Fig. 6C). These miRNAs in E25T-2D could together induce the osteogenic differentiation of BMSCs and angiogenic differentiation of HUVECs by interacting with their targets, such as CREB binding protein (CREBBP), dickkopf1 (DKK1), thrombospondin 1 (TSP1), kruppel like factor 2 (KLF2), and Claudin-11. ${ }^{26,27}$ In addition, in E-25T-2D, antiinflammatory miRNAs, such as mmu-miR-125a-5p, mmu-miR-130b-5p, mmu-miR-181a-5p and mmu-miR146b-5p, showed upregulated expression (Fig. 6D). 

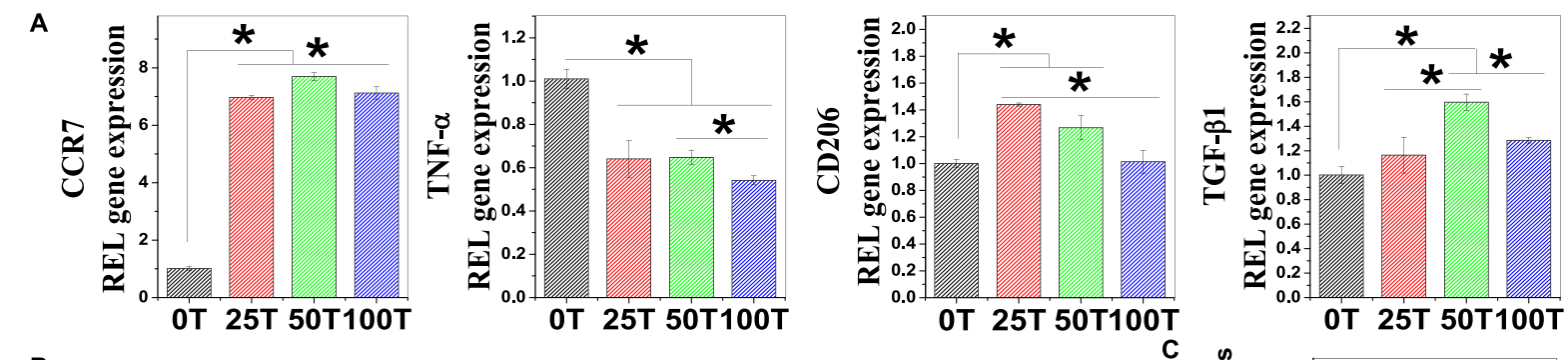

B
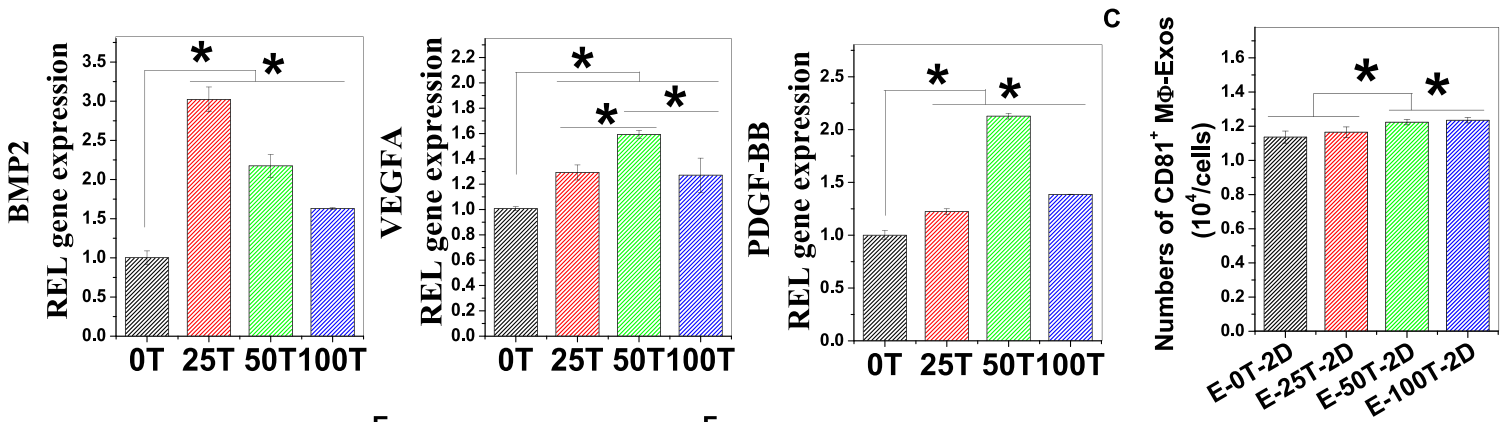

D
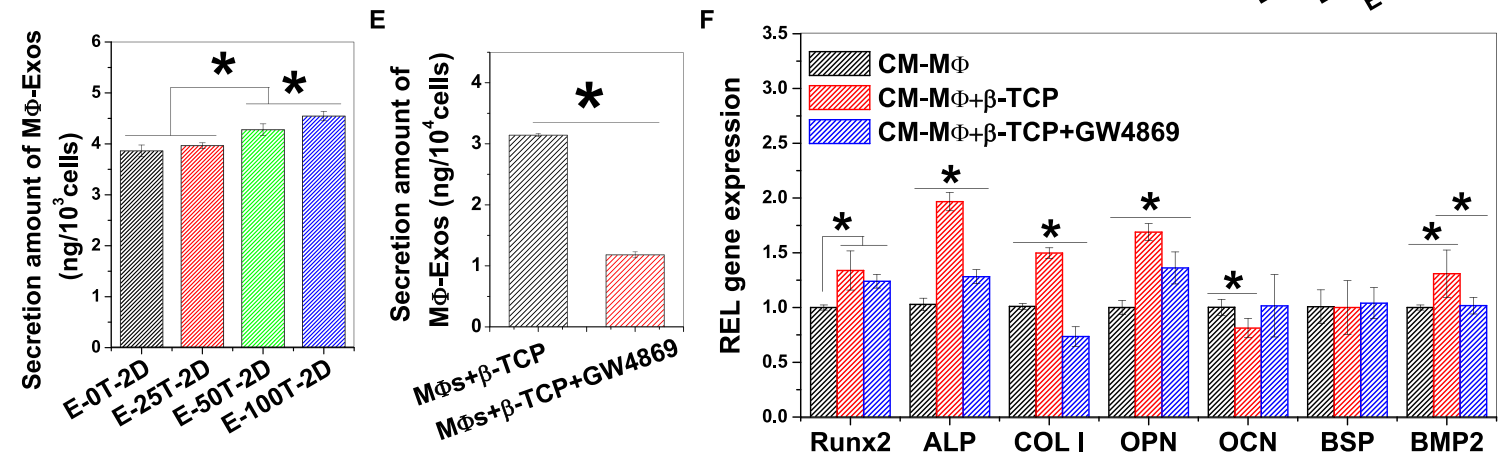

G
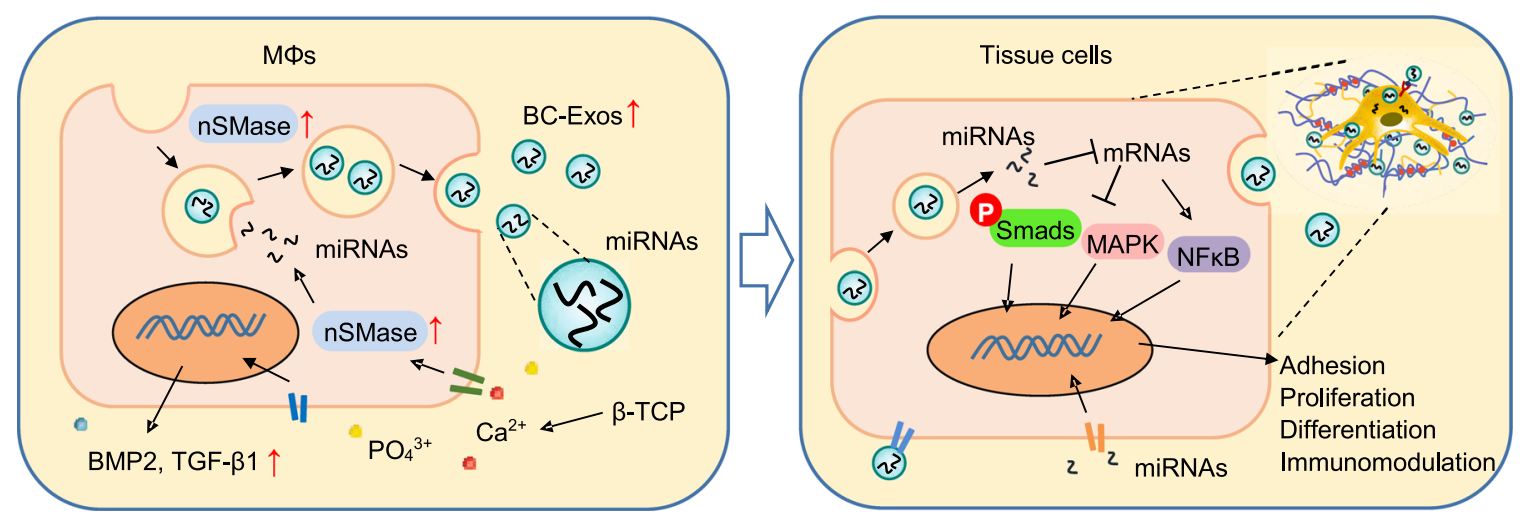

Fig. $7 \beta$-TCP stimulation modulated MФ polarization and exosome production. A Effect of $\beta$-TCP extracts on MФs polarization. MФs stimulated by $\beta$-TCP extracts for 2 days were Mix-type with upregulated expression of CCR7, CD206, and TGF- $\beta 1$ and downregulated expression of TNF- $\alpha$ ( $n=3$, $\left.{ }^{*} p<0.05\right)$. B The expression of BMP2, VEGFA, and PDGF-BB was upregulated in $\beta$-TCP-stimulated MФs $\left(n=3,{ }^{*} p<0.05\right)$. MФ-Exo production detected by $\mathbf{C}$ ExoELISA-ULTRA CD81 assays and $\mathbf{D}$ BCA protein assays $\left(n=3,{ }^{*} p<0.05\right)$. MФ-Exos induced by $\beta$-TCP extracts of $0,25,50$, and $100 \mathrm{mg} / \mathrm{mL}$ for 2 days were referred to as E-0T-2D, E-25T-2D, E-50T-2D, and E-100T-2D, respectively. E Effect of the inhibitor GW4869 on MD-exosomal protein secretion $\left(n=3,{ }^{*} p<0.05\right)$. $\mathbf{F}$ Effect of the inhibitor GW4869 on the paracrine effect of $\beta$-TCP-stimulated MФs on BMSCs at Day 3. Gene expression of Runx2, ALP, COL I, OPN, and BMP2 was significantly impaired in the CM-MФs+ $\beta-T C P+$ GW4869 group compared to the CM- MФs+ $\beta-T C P$ group $\left(n=4,{ }^{*} p<0.05\right)$. G Summary of the possible mechanism by which $\beta$-TCP regulates the immunomodulation of macrophages and M $\Phi$-Exo-mediated paracrine effects: $\beta$-TCP stimulation-induced changes in the cell microenvironment, such as ionic concentrations of $\mathrm{Ca}^{2+}$ and $\mathrm{PO}_{4}{ }^{3-}$, which resulted in the increased activity of nSMases to promote BC-Exo production and alter exosomal miRNA cargos. Exosomal miRNAs could contribute to the improved adhesion, proliferation, differentiation, and immunomodulation of tissue cells by signaling pathways, such as the MAPK and NFKB pathways. 
Enhanced miR-125a-5p expression was proven to suppress the classic activation of macrophages by targeting toll-like receptor (TLR) 2 and TLR4. ${ }^{28}$ Furthermore, proinflammatory miRNAs, such as mmu-miR-5126, mmu-miR-3473e, mmu-miR-671-5p, and mmu-miR-1555p, showed obviously downregulated expression (Fig. 6D). MiR-3473e is one of the specific miRNAs of M1-type MФs in the early response, and the inhibition of miR-671$5 p$ could enhance the anti-inflammatory ability of stem cells against LPS-induced extensive acute lung injury by upregulating IL-1ra and soluble tumor necrosis factor receptor II (sTNFRII) expression. ${ }^{29,30}$ BC-Exos may elicit a modulatory effect on the macrophage-mediated inflammatory response by regulating the TLR/tumor necrosis factor receptor-related factor 6 (TRAF6)/nuclear factor $\mathrm{k}-\mathrm{B}(\mathrm{NF} \mathrm{B})$ pathways via exosomal miRNAs. Overall, macrophages responded to $\beta$-TCP stimulation by releasing exosomes containing highly specific miRNA cargos, and alterations in exosomal miRNAs may play important roles in regulating the immunomodulation, osteogenesis, and angiogenesis mediated by 3D-printed BC-Exos.

\section{$\beta$-TCP stimulation modulated MФ polarization and exosome production}

The sorting of specific miRNAs to exosomes is the result of parental cells responding to external stimuli. Thus, the activity and phenotype of macrophages were explored further. $\beta$-TCP extracts promoted MФ proliferation after 3 days, and the extract concentration had little effect on cell survival and morphology (Fig. S13A $-C)$. Fluorescence images of macrophage morphology in Fig. S13B show that the unstimulated macrophages (0T) were round without pseudopodia, presenting an unpolarized state, namely, M0 status. The МФs stimulated with $\beta$-TCP extracts for 2 days upregulated the expression of the proinflammatory genes IL6 and chemokine receptor 7 (CCR7) and the anti-inflammatory genes mannose receptor (CD206) and TGF- $\beta 1$ while downregulating the expression of the anti-inflammatory genes IL10 and Arg1 and the proinflammatory gene TNF- $\alpha$ (Fig. 7A and Fig. S13D). These cells exhibited a "function-actively" mixed phenotype (Mix-type) rather than a fully polarized M1 or M2-type phenotype. M1 and M2 signatures often coexist depending on the balance of stimulatory and inhibitory activities and the tissue microenvironment. In addition, Fig. 7B shows that the expression of BMP2, VEGFA, and platelet-derived growth factor BB (PDGF-BB), which are conducive to osteogenesis and angiogenesis, was significantly upregulated in the $\beta$-TCP-stimulated macrophages. Our previous study showed that the $\beta$-TCPstimulated macrophages promoted the osteogenic differentiation of BMSCs via the BMP2 signaling pathway. ${ }^{31}$ In addition to the phenotypic switch, the difference in exosome levels between the unstimulated and stimulated macrophages was detected via ExoELISA-ULTRA CD81 assays and BCA protein assays. The results in Fig. 7C, D show that $\beta$-TCP-stimulating macrophages produced more exosomes at the particle number and protein levels. $\mathrm{M} \Phi$-Exo production increased with increasing $\beta$-TCP extract concentration. Studies have shown that several key proteins and signaling pathways could strikingly affect exosome biogenesis, including the family of neutral sphingomyelinases (nSMases) and the Rab family of small GTPases. ${ }^{32,33}$ It has been reported that the activation of nSMases, such as $\mathrm{Ca}^{2+}$-dependent nSMase2, cytosolic $\mathrm{Mg}^{2+}$-independent nSMase, and cytosolic $\mathrm{Zn}^{2+}$-dependent acidic SMase, is ion-dependent. ${ }^{34}$ The dissolution of bioceramic powders likely leads to changes in the cell microenvironment, such as ionic concentrations, to induce the activation of nSMases for exosome production (Table S4). A previous study showed that the ion products from 45S5 Bioglass enhanced the production of MSCderived exosomes by upregulating the expression of nSMase 2 and Rab27a. ${ }^{33}$ In addition, nSMase 2 was the first molecule reported to be related to miRNA secretion into exosomes. ${ }^{35}$ This finding suggests that nSMases may participate in $\beta$-TCP-induced $M \Phi$-Exo production and exosomal miRNA sorting. GW4869 is an inhibitor of nSMase 2 and can result in the reduced secretion of exosomes and miRNAs through inhibition of ceramide generation by nSMase $2 .{ }^{36}$ Pretreatment with $10 \mu \mathrm{M} \mathrm{GW} 4869$ decreased $M \Phi$-exosomal protein secretion to $\sim 38 \%$ (Fig. $7 \mathrm{E})$. Conditioned medium from the $\beta$-TCP-stimulated macrophages with GW4869 (referred to as CM-MФ+ $\beta-\mathrm{TCP}+\mathrm{GW} 4869$ ) diminished the osteogenic differentiation of BMSCs by suppressing the expression of osteogenic genes, such as ALP, COL I, OPN, and BMP2 (Fig. 7F). This result indicates the roles of MФ-Exos and exosomal miRNAs in $\beta$-TCP-mediated osteogenesis. Moreover, the improved osteogenic differentiation of BMSCs induced by $\beta$-TCP-stimulating macrophages reveals the importance of bioceramics in regulating cellto-cell communication. $\beta$-TCP bioceramics modulated the gene expression, exosome production, and exosomal miRNA cargos of macrophages, leading to an effect of MФs and BC-Exos on immunomodulation and osteogenesis/angiogenesis (Fig. 7G).

The macrophage phenotype presents dynamic characteristics in response to the tissue microenvironment, thereby influencing the functionality of $M \Phi$-Exos. ${ }^{12,33}$ After the $\beta$-TCP stimulation time was prolonged to 4 days, МФs polarized toward an anti-inflammatory phenotype (M2type) with increased expression of anti-inflammatory genes (TGF- $\beta 1$, Arg1, IL10) and decreased expression of proinflammatory genes (CCR7, IL6, TNF- $\alpha$ ) (Fig. S13E). Comparing the effects of BC-Exos derived from M2-type MФs with BC-Exos from Mix-type MФs on immunomodulation, 
osteogenesis, and angiogenesis, we found that BC-Exos all had a distinct effect on the immunomodulation of macrophages, and the BC-Exos from M2-type MФs exhibited a weaker ability to enhance osteogenesis and a comparable ability to the BC-Exos from Mix-type MФs in promoting angiogenesis. Figure S14 shows that scaffolds with BC-Exos from M2-type MФs downregulated the expression of iNOS2, IL6, and TNF- $\alpha$ in MФs, regulated HUVEC migration, and impaired BMSC chemotaxis mediated by MФs. The osteogenic differentiation of BMSCs on scaffolds with BC-Exos from M2-type MФs was also decreased with lower expression of ALP, Runx2, COL I, BSP, OCN, and BMP2, although the proliferation, adhesion, and expression of GAL- 1 and TSG- 6 of BMSCs on scaffolds were improved (Fig. S15). Regarding angiogenesis, HUVECs cultured with BC-Exos from M2-type MФs exhibited higher expression of VEGFA, vWF, HIF- $1 \alpha$, and bFGF2 and more tube formation (Fig. S16). Three-dimensional-printed BC-Exos from M2-type MФs also promoted HUVEC proliferation, adhesion, and the expression of some genes related to angiogenesis (Fig. S17). In summary, the different polarizations of macrophages in response to stimulation with $\beta$-TCP indeed affected the biological functionality of BC-Exos, especially the effect on osteogenesis. Research has shown that M1Exos, rather than M0-Exos or M2-Exos, support the osteogenic differentiation of MSCs. ${ }^{37}$ Exosomes derived from LPS-stimulated monocytes increased the gene expression of Runx2, BMP-2, and matrix metalloproteinases for tissue remodeling. ${ }^{38,39}$ However, there are also opposite results: M0- and M2-EVs promote bone repair and regeneration, while M1-EVs inhibit bone repair. ${ }^{10,40}$ The exact role of exosomes derived from differently polarized MФs in osteogenesis has not been conclusively determined. This discrepancy can be due to the differences in culture conditions and cell lineage. In addition, unpolarized macrophage-generated exosomes were found to have biological effects on osteogenesis, angiogenesis, and inflammation. The possible reason may be due to the various bioactive substances, such as proteins, nucleic acids, and lipids contained in E-0T-2D. The results of exosomal miRNA sequencing revealed that E-0T-2D had miRNAs that can regulate osteogenesis, angiogenesis, and inflammation, such as the pro-osteogenic miRNA mmu-miR-690, the proangiogenic miRNAs mmu-let-7d-5p and mmu-miR25-3p, and the anti-inflammatory miRNAs mmu-miR146a-5p, miR-330-5p and mmu-miR-423-5p. MiR-690 has been identified as a positive regulator of Runx2-induced osteogenic differentiation of $\mathrm{C} 2 \mathrm{C} 12$ cells. ${ }^{41} \mathrm{MiR}-25-3 \mathrm{p}$ can promote vascular permeability and angiogenesis by regulating the expression of VEGFR2 and Claudin 5 in endothelial cells. ${ }^{42}$ Overexpression of miR-330-5p restrained inflammation and oxidative stress induced by lipopolysaccharides (LPS) by targeting TRAF6 to suppress the activation of NF- $\mathrm{KB}$ signaling. ${ }^{43}$ These results suggested that unpolarized macrophage-generated exosomes may modulate immunomodulation and osteogenesis/angiogenesis through exosomal miRNAs. Similar results were found in the study of Xia et al., ${ }^{37}$ in which exosomes from unpolarized MФs increased the ALP activity of BMMSCs and downregulated the expression of chondrogenesisrelated genes.

Overall, it was confirmed that 3D printing of BC-Exos has an exciting effect on immunomodulation and osteogenesis/ angiogenesis. $\beta$-TCP bioceramics may induce changes in the cell microenvironment, leading to $M \Phi$ polarization with alterations in gene expression, including mRNA and miRNA expression, and increased exosome production to further adjust cell-to-cell communication in tissue regeneration. However, the possible mechanism by which $\beta$-TCP regulates exosomal miRNA sorting still requires intensive study. In addition, research has shown that immune cells can apply exosomes to activate the immune system and enhance immune responses upon triggering by danger signals. ${ }^{2}$ Whether $3 \mathrm{D}$ printing of $\mathrm{M} \Phi$-Exos would induce any systemic immunogenicity or off-target effects is not clear. Hence, the local or systemic immune reaction elicited by the 3D printing of $\mathrm{BC}$-Exos in vivo and the bone repair of BC-Exo scaffolds will be evaluated in future work.

\section{Conclusions}

Three-dimensional-printed scaffolds consisting of bioceramic-induced macrophage exosomes were designed in this work. The potential roles of BC-Exos in regulating immunomodulation, osteogenesis, and angiogenesis were demonstrated. Through sustained release, 3D-printed BCExos induced the immunomodulation of macrophages, osteogenic differentiation of BMSCs, and vascular differentiation of HUVECs by upregulating the expression of genes related to immunogenesis, osteogenesis, and angiogenesis. The possible mechanism is that $\beta$-TCP stimulation regulated macrophage polarization and miRNA cargos in $\mathrm{BC}$-Exos and thus triggered the immunomodulation and osteogenic and angiogenic differentiation pathways in tissue cells. This study reveals the paracrine effects of macrophages on immune and bone-related cells via biomaterial-induced exosomes. The design concept of cell-free 3D printing scaffolds with exosomes derived from biomaterial-activated macrophages increases the applications of immune cellderived exosomes for tissue regeneration and proposes an innovative direction for the design of bone regeneration and repair biomaterials with immunomodulation.

\section{Acknowledgements}

This work was supported by the National Key Research and Development Program of China (2018YFC1105201), Innovation Cross Team of Chinese

Academy Sciences (JCTD-2018-13), Science and Technology Commission of

Shanghai Municipality (20442420300, 21DZ1205600), and Shanghai Pujiang Talent Program (2020PJD080). 


\section{Author contributions}

Y.S. and C.W. conceived the idea. C.W. supervised the execution of the whole work. Y.S. explained the experimental results. Y.S. and B.Z. performed the experiments and image analysis. Y.S. designed the samples. Y.S. and D.Z. fabricated the samples. Y.S. wrote the paper. All authors were involved in the discussion of the results and commented on the paper.

\section{Competing interests}

The authors declare no competing interests.

\section{Publisher's note}

Springer Nature remains neutral with regard to jurisdictional claims in published maps and institutional affiliations.

Supplementary information The online version contains supplementary material available at https://doi.org/10.1038/s41427-021-00340-w.

Received: 13 April 2021 Revised: 2 October 2021 Accepted: 6 October 2021. Published online: 19 November 2021

\section{References}

1. Song, Y. et al. Localized injection of miRNA-21-enriched extracellular vesicles effectively restores cardiac function after myocardial infarction. Theranostics $\mathbf{9}$ 2346-60 (2019).

2. Veerman, R. E., Gucluler Akpinar, G., Eldh, M. \& Gabrielsson, S. Immune cellderived extracellular vesicles-functions and therapeutic applications. Trends Mol. Med. 25, 382-94 (2019).

3. Zhu, X. et al. Comprehensive toxicity and immunogenicity studies reveal minimal effects in mice following sustained dosing of extracellular vesicles derived from HEK293T cells. J. Extracell. Vesicles 6, 1324730 (2017).

4. Imai, T. et al. Macrophage-dependent clearance of systemically administered B16BL6-derived exosomes from the blood circulation in mice. J. Extracell. vesicles 4, 26238 (2015).

5. Zhang, K. et al. Enhanced therapeutic effects of mesenchymal stem cellderived exosomes with an injectable hydrogel for hindlimb ischemia treatment. ACS Appl Mater. Interfaces 18, 30081-91 (2018).

6. Li, J., Wu, C., Chu, P. K. \& Gelinsky, M. 3D printing of hydrogels: rational design strategies and emerging biomedical applications. Mater. Sci. Eng.: R: Rep. 140, $100543(2020)$

7. Gao, M. et al. Exosomes-the enigmatic regulators of bone homeostasis. Bone Res. 6, 36 (2018)

8. Narayanan, R., Huang, C. C. \& Ravindran, S. Hijacking the cellular mail: exosome mediated differentiation of mesenchymal stem cells. Stem Cells Int. 2016 3808674 (2016)

9. Wang, Z. et al. Switching on and off macrophages by a "Bridge-Burning" coating improves bone-implant integration under osteoporosis. Adv. Funct. Mater. 31, 2007408 (2020).

10. Yan, W. et al. M2 macrophage-derived exosomes promote the c-KIT phenotype of vascular smooth muscle cells during vascular tissue repair after intravascular stent implantation. Theranostics 10, 10712-10728 (2020).

11. Kang, M. et al. Bone regeneration is mediated by macrophage extracellular vesicles. Bone 141, 115627 (2020)

12. Lebaudy, E., Fournel, S., Lavalle, P., Vrana, N. E., \& Gribova, V. Recent advances in antiinflammatory material design. Adv. Healthcare Mater. 10, 2001373 (2020).

13. Chen, Z. et al. Osteoimmunomodulation for the development of advanced bone biomaterials. Mater. Today 19, 304-321 (2016).

14. Sun, Y. et al. Biological responses to M13 bacteriophage modified titanium surfaces in vitro. Acta Biomater. 58, 527-538 (2017).

15. Li, M. et al. Macrophage-derived exosomes accelerate wound healing through their anti-inflammation effects in a diabetic rat model. Artif. Cells, Nanomed. Biotechnol. 47, 3793-3803 (2019).

16. Vasconcelos, D. P., Aguas, A. P., Barbosa, M. A., Pelegrin, P. \& Barbosa, J. N. The inflammasome in host response to biomaterials: bridging inflammation and tissue regeneration. Acta Biomater. 83, 1-12 (2019).

17. Salcedo, R. et al. Human endothelial cells express CCR2 and respond to MCP-1: direct role of MCP-1 in angiogenesis and tumor progression. Blood 96, 34-40 (2000).
18. Fu, Q. et al. Primary tumor-derived exosomes facilitate metastasis by regulating adhesion of circulating tumor cells via SMAD3 in liver cancer. Oncogene $\mathbf{3 7}$ 6105-18 (2018).

19. Sioud, M., Mobergslien, A., Boudabous, A. \& Fløisand, Y. Mesenchymal stem cell-mediated $T$ cell suppression occurs through secreted galectins. Int $\mathrm{J}$. Oncol. 38, 385-390 (2011).

20. Song, W.-J. et al. TSG-6 released from intraperitoneally injected canine adipose tissue-derived mesenchymal stem cells ameliorate inflammatory bowel disease by inducing M2 macrophage switch in mice. Stem Cell Res. Ther. 9, 91 (2018).

21. Yang, X. et al. Callus mineralization and maturation are delayed during fracture healing in interleukin-6 knockout mice. Bone 41, 928-936 (2007).

22. Deng, W. et al. Mesenchymal stem cells promote CD206 expression and phagocytic activity of macrophages through IL-6 in systemic lupus erythematosus. Clin. Immunol. 161, 209-216 (2015).

23. Shi, Y. et al. How mesenchymal stem cells interact with tissue immune responses. Trends Immunol. 33, 136-143 (2012).

24. Franco, C. A. et al. SRF selectively controls tip cell invasive behavior in angiogenesis. Development 140, 2321-2333 (2013).

25. Zhai, M., Zhu, Y., Yang, M. \& Mao, C. Human mesenchymal stem cell derived exosomes enhance cell-free bone regeneration by altering their miRNAs profiles. Adv. Sci. 7, 2001334 (2020).

26. Zheng, R. et al. Upregulated microRNA-330-3p promotes calcification in the bicuspid aortic valve via targeting CREBBP. Mol. Med. Rep. 22, 2351-2363 (2020).

27. Yang, S., Guo, S., Tong, S., Sun, X. Exosomal miR-130a-3p regulates osteogenic differentiation of human adipose-derived stem cells through mediating SIRT7/ Wnt/B-catenin axis. Cell Prolif. 53, e12890 (2020).

28. Wang, W. \& Guo, Z.H. Downregulation of IncRNA NEAT1 ameliorates LPSinduced inflammatory responses by promoting macrophage M2 polarization via miR-125a-5p/TRAF6/TAK1 axis. Inflammation 43, 1548-1560 (2020).

29. $L u$, L. et al. Time Series miRNA-mRNA integrated analysis reveals critical miRNAs and targets in macrophage polarization. Sci. Rep. 6, 37446 (2016).

30. Lien, G.S. et al. The ability to suppress macrophage-mediated inflammation in orbital fat stem cells is controlled by miR-671-5p. Stem Cell Res. Ther. 5, 97 (2014).

31. Chen, Z. et al. Osteogenic differentiation of bone marrow MSCs by betatricalcium phosphate stimulating macrophages via BMP2 signalling pathway. Biomaterials 35, 1507-1518 (2014).

32. Trajkovic, K. et al. Ceramide triggers budding of exosome vesicles into multivesicular endosomes. Science 319, 1244-1247 (2008).

33. $\mathrm{Wu}, \mathrm{Z}$., He, D. \& Li, H. Bioglass enhances the production of exosomes and improves their capability of promoting vascularization. Bioact. Mater. 6 , 823-835 (2021).

34. Kim, S. K. et al. Purification of neutral sphingomyelinase 2 from bovine brain and its calcium-dependent activation. J. Neurochem. 112, 1088-1097 (2010).

35. Zhang, J. et al. Exosome and exosomal microRNA: trafficking, sorting, and function.Genomics, Proteomics Bioinformatics 13, 17-24 (2015).

36. Kosaka, N. et al. Secretory mechanisms and intercellular transfer of microRNAs in living cells. J. Biol. Chem. 285, 17442-17452 (2010).

37. Xia, Y. et al. Exosomes derived from $\mathrm{MO}, \mathrm{M} 1$, and $\mathrm{M} 2$ macrophages exert distinct influences on the proliferation and differentiation of mesenchymal stem cells. PeerJ 8, e8970 (2020).

38. Rameshwar, P. et al. Monocyte exosomes stimulate the osteogenic gene expression of mesenchymal stem cells. PLoS One 8, e75227 (2013).

39. Gebraad, A. et al. Monocyte-derived extracellular vesicles stimulate cytokine secretion and gene expression of matrix metalloproteinases by mesenchymal stem/stromal cells. FEBS J. 285, 2337-2359 (2018).

40. Liu, A. et al. Macrophage-derived small extracellular vesicles promote biomimetic mineralized collagen-mediated endogenous bone regeneration. Int. J. Oral Sci. 12, 33 (2020).

41. Yu, S. et al. MiR-690, a Runx2-targeted miRNA, regulates osteogenic differentiation of $\mathrm{C} 2 \mathrm{C} 12$ myogenic progenitor cells by targeting NF-kappaB p65. Cell Biosci. 6, 10 (2016)

42. Zeng, Z. et al. Cancer-derived exosomal miR-25-3p promotes pre-metastatic niche formation by inducing vascular permeability and angiogenesis. Nat Commun. 9, 5395 (2018).

43. Xing, P.-C., An, P., Hu, G.-Y., Wang, D.-L. \& Zhou, M.-J. LncRNA MIAT promotes inflammation and oxidative stress in sepsis-induced cardiac injury by targeting miR-330-5p/TRAF6/NF-kB axis. Biochem. Genet. 58, 783-800 (2020). 